\title{
SPACED SEEDS FOR CROSS-SPECIES CDNA-TO-GENOME SEQUENCE ALIGNMENT*
}

\author{
LEMING ZHOU ${ }^{\dagger}$, INGRID MIHAI ${ }^{\ddagger}$, AND LILIANA FLOREA ${ }^{\S}$
}

\begin{abstract}
We review recent developments in spaced seed design for cross-species sequence alignment. We start with a brief overview of original ideas and early techniques, and then focus on more recent work on finding accurate (sensitive and specific) seeds for cross-species cDNA-to-genome alignment. These recent developments include methods and models for estimating seed specificity and determining sensitive and specific seeds, finding seeds that can be applied to a wide range of comparisons, and applying seed models to other computational biology areas, such as gene finding.
\end{abstract}

Keywords: cDNA, Genome, Spaced Seeds, Markov Model

1. Introduction. New high-throughput and cost-effective technologies have revolutionized our ability to sequence complex organisms, and are expected to lead to a significant increase in the number of available genomes for species from all branches of life [1]. The first and most important step in analyzing these genomes is gene annotation, that is, accurately identifying the locations and exon-intron structures of genes along the genome, and further determining their function.

There are two primary classes of methods for identifying genes in a given genomic sequence. The first class, ab initio methods (GenScan [2], Genie [3], GeneMark [4], FGenesH [5]), use machine-learning techniques to analyze a single genomic sequence and predict the locations of genes. Such methods are reasonably accurate at finding coding exons, but are not effective at detecting untranslated regions (UTRs) and alternatively spliced or overlapping genes [6].

The second class, comparative methods, predict exons based on sequence similarity of protein or expressed DNA (cDNA, EST, mRNA) with genomic sequences containing those genes. These methods are the most reliable for inferring the gene structure, and thus genome annotation projects have routinely used cDNA sequences from the same species to annotate genes. Although several projects exist that produce fulllength cDNA sequences [7-9], they focus on a handful of high-priority species, such as human, mouse, rat, cow and zebrafish. For most newly sequenced species, few native cDNA sequences are available in the databases. Consequently, gene annotation

\footnotetext{
${ }^{*}$ Dedicated to Michael Waterman on the occasion of his 67 th birthday.

$\dagger$ Department of Health Information Management and McGowan Institute for Regenerative Medicine, University of Pittsburgh, Pittsburgh, PA 15260, USA. E-mail: lzhou1@pitt.edu, corresponding author.

${ }^{\ddagger}$ Department of Computer Science, George Washington University, Washington DC, 20052, USA. E-mail: ingrid@gwu.edu

$\S$ Center for Bioinformatics and Computational Biology, University of Maryland, College Park, MD 20742, USA. E-mail: florea@umiacs.umd.edu, corresponding author.
} 
projects for these genomes will need to rely instead on cDNA information from other species. While several tools exist that map cDNA sequences to the genome of the same species accurately and efficiently ( $\operatorname{sim} 4$ [10], Spidey [11], BLAT [12], GMAP [13], MGAlignIt [14], ESTmapper [6]), their performance drops in cross-species comparisons, as the sequence similarity decreases. Furthermore, tools specialized for cross-species comparisons (EXALIN [15], GeneSeqer [16], EST_GENOME [17]) lack the speed required for whole-genome annotation and are still very limited in the evolutionary range they address. Therefore, there is a critical need for alignment tools for mapping gene sequences to the genome of a related species accurately and efficiently.

The first and foremost limitation in aligning cross-species is the sensitivity of alignment, in which the choice of seed is the most important factor. A seed is a match pattern used to detect local alignments. For instance, blast $[18,19]$ searches for exact word matches of length 11 between the two sequences, which it later extends to local alignments by allowing for gaps and substitutions. Such match pattern, or seed, is called continuous, and can be represented as a pattern of 1s (11111111111), where each 1 represents a position required to match. The blast seed and its length variations have served as match patterns for many generations of alignment programs, until its limitations have recently been revealed. Indeed, the blast seed loses sensitivity as the differences between the sequences increase with the evolutionary distance between the compared species. Reducing the size of the seed increases its ability to detect weaker similarities, however at a considerable loss in specificity.

Spaced seeds were recently introduced to increase alignment sensitivity with a relatively small change in specificity. Unlike continuous seeds, spaced seeds allow for some wildcard positions in the pattern, marked with 0s. For instance, the seed 11011011011011011 intuitively takes into account the weaker matching pattern at the third (wobble) position in the codon to increase the success rate when mapping ESTs or genes to target genomic sequences [20]. The length of the seed pattern is called span, and the number of $1 \mathrm{~s}$ in the seed is the weight of the seed. The definition of weight may change for different classes of seeds, as we will see in later sections. For the same weight, spaced seeds were shown to be more sensitive than continuous seeds in most practical cases [21]. As many $\{0,1\}$ seeds can be formed, even while maintaining the weight constant, a natural question that arises is: Given an alignment pattern, what is the best, or most sensitive, seed? Ma et al. [22] formally introduced spaced seeds and proposed a mathematical framework for estimating the sensitivity of a seed [23], which allowed them to determine optimal seeds for a class of alignment applications. Refinements and extensions of their model to other classes of alignment were soon proposed, and are reviewed in the following sections.

While sensitivity has been in the cross-hairs of seed design efforts, specificity has received considerably less attention. Whereas sensitivity reflects the ability of a seed to detect biologically meaningful sequence similarities, specificity measures the ability 
of the seed to differentiate between true and spurious matches, and directly affects the outcome as well as the speed of processing. We recently gave a rigorous formulation for specificity for the case of cDNA-to-genome alignment and suggested strategies for selecting accurate seeds that are both sensitive and specific [24].

As the field of seed design is maturing, new questions and challenges emerge from their application to practical alignment tools and to other computational biology areas. So far seed theory has focused on designing good seeds for one comparison at a time, usually using human-mouse as the model of interest. As the number of sequenced species increases, however, designing seeds for each individual comparison quickly becomes prohibitive, given both the computational time involved and the increased complexity when selecting the appropriate program parameters for a type of comparison. A natural question that arises is: Are there seeds that can be used by multiple comparisons, and under what conditions? We gave a preliminary characterization of both such seeds, which we call universal [25], and conditions [26], which we review here.

The remainder of the paper is organized as follows: Section 2 describes the early introduction of spaced seeds and more recent refinements and extensions, and the application of seeds to alignment programs; Section 3 illustrates the general seed design framework in the context of our work with determining accurate (i.e., sensitive and specific) spaced seeds for cDNA-to-genome comparison; Section 4 presents new directions in seed design, including our ongoing work with characterizing and determining universal spaced seeds, and exploring the potential of using spaced seeds for gene detection from sequence alignments. Lastly, Section 5 summarizes the results.

\section{Background and Previous Work.}

2.1. Intuitive Spaced Seeds. "Seeds" are match patterns used to locate regions of similarity between two sequences. The first and most enduring seed pattern was 11111111111, requiring an exact match of 11 contiguous bases. First introduced in blast $[18,19]$, it was later implemented, with length variations, in most alignment programs that succeeded it. Only recently spaced seeds were introduced, which allow for a number of wildcard positions in the pattern, marked with 0s. Spaced seeds were informally used in programs such as WABA [20], a cDNA-to-genome alignment program, where every third position was omitted from the comparison (11011011011011011), consistently with the weaker match at the wobble codon position.

2.2. Formal Spaced Seeds Framework. Ma and co-authors formally introduced the concept of spaced seeds in [22] and proposed a mathematical framework for estimating the sensitivity of a seed given an alignment model and determining optimal seeds [23]. Their framework consists of two components: a seed model, originally a $\{0,1\}$ pattern as described above, and an alignment model. In a seed, 1s indicate match positions and 0s indicate wildcard positions. Alignments are also represented 


\section{ACGCACCAACCACTTCAGTCCAGT ACCTACCGACCACCTCAGCGAAGT \\ Alignment \\ Convert to $\{0,1, x\}$ string}

\section{0x111x11111×1111x00111 Alignment \\ $1101 \times 111 \times 1011$ \\ Seed match

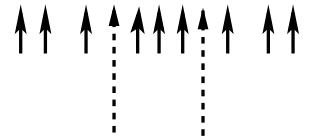 \\ Match-only positions \\ Match or transition positions}

Fig. 1. Seed detection of an alignment.

as $\{0,1\}$ strings generated according to a model, for instance a Bernoulli chain, where 1 s represent matches and 0s now indicate mismatches. With this notation, the sensitivity of a seed is defined formally as the probability that the seed will detect an alignment string of fixed length $L$ generated by the model (Figure 1). An optimal seed is by definition a seed with the highest sensitivity.

In the Bernoulli alignment model of [23], alignment positions could be independently 0 or 1 with probabilities $p$ and $1-p$, respectively, where $p$ is inferred from the overall level of sequence similarity in the alignment. At the time, such model was appropriate to describe alignments of large genomic sequences, which can be seen as random. Subsequent studies evaluated increasingly complex alignment models for this and other alignment applications.

Soon thereafter, Buhler et al. [21,27] developed an order 1 alignment Markov model to investigate coding and non-coding regions. A Markov model of order $m$ assumes that the 0 or 1 symbol at any position depends on the symbols at the preceding $m$ positions. Later, Brejová et al. [28] proposed two extensions of the Bernoulli model for alignments within coding regions. In the $M(3)$ model, the probability for a position to be a match or mismatch depends on its relative location within a codon, and positions in the alignment are independent. In the $M(8)$ model, multiple codons are considered, each with its own conservation pattern.

In a parallel development, to further improve the seed sensitivity over $\{0,1\}$ seeds, transition-only wildcards, marked with $x$ or \#, were introduced to differentiate between transitions and transversions, first implemented in blastz [29]. Later, Noe and

Kucherov [30] formalized the concept and showed that spaced seeds with transitions extend the sensitivity range of $\{0,1\}$ seeds, while Zhou and Florea [24] additionally provided a framework for calculating seed specificity and showed that transition spaced seeds achieve better sensitivity-specificity tradeoffs in practice. 
2.2.1. Methods for Calculating Seed Sensitivity. The following two questions are at the heart of seed design efforts: i) What is the sensitivity of a seed given a model of alignment? and ii) What is the best seed for that model?

To answer the first question, Keich et al. [23] gave a dynamic programming algorithm for calculating seed sensitivity recursively, and Choi and Zhang [31] provided a similar alternative. A more popular method was that proposed in [21], which used tries and the Aho-Corasick algorithm [32] to construct a minimal Deterministic Finite Automaton (DFA) recognizing all possible matches of the seed and to calculate their probability, or equivalently the seed sensitivity, recursively. More recently, Kucherov et al. $[33,34]$ proposed a generalized framework for estimating the sensitivity of several types of spaced seeds. Instead of the two components in the traditional framework, namely a seed model and an alignment model, their framework uses three: the target alignments, a probability distribution of the alignments, and a seed model. This formulation allows more efficient automata to be built, which speeds up the seed sensitivity calculation.

To determine optimal seeds, in early work Keich et al. [23] determined optimal spaced seeds by exhaustive search, calculating each seed's sensitivity recursively. Such a comprehensive approach was feasible given the relatively small seed space $(11,440$ seeds with weight 11 and span 18). As the space and complexity of the search increased with larger seed spans and more sophisticated alignment models, heuristic solutions were developed for optimizing seeds, which were shown to produce close approximations that worked well in practice. Among the most popular, a hill-climbing heuristic algorithm was proposed in $[21,24]$ to speed up the search process. The algorithm starts from a random seed and swaps any two distinct symbols with the goal to optimize the sensitivity locally. If a better seed is found, it becomes the start seed for variations in the next cycle, until there is no improvement to the seed sensitivity. The procedure is restarted multiple times, to avoid convergence to a local optimum. In [35], the authors exploit the structure of observed optimal seeds to derive rules for pruning the search space, which they later refine in [36]. Lastly, an alternative to the definition of seed sensitivity that is easier to compute and thus can be used to design large seeds was introduced in [37]. It uses the overlap complexity of seeds as a surrogate for sensitivity. This method, however, is only applicable to genomic sequence alignment represented by Bernoulli models.

2.3. Generalized Spaced Seeds. Several variations of the traditional seed design model have been proposed, to further increase sensitivity. For instance, carefully selected multiple seeds can be simultaneously used to improve alignment discovery. Their set of hits is the union of the hits found by each seed. The formalism of multiple seeds $[38,39]$ is a relatively straightforward extension of the single-seed models.

One generalization of spaced seeds that can also be applied to protein alignment 
is that of vector seeds [40]. Vector seeds model an alignment not as $\{0,1\}$ strings, but as sequences of real numbers. Accordingly, each position in the seed pattern is a real value representing the weight of a match or substitution at that position in the total match score. A seed match is declared when the total score exceeds an a priori fixed threshold.

Both multiple spaced seeds and vector seeds can further improve the sensitivity over single spaced seeds, but they increase the memory and running time of searches, both of which are critical for practical high-throughput applications.

2.4. Applications of Spaced Seeds to Alignment Programs. Since their introduction in 2002, mathematically optimized spaced seeds have been rapidly adopted by alignment programs. Optimal spaced seeds obtained in $[22,23]$ were implemented in the programs PatternHunter [22] and blastz [29]. As a variation intended to further increase seed sensitivity, blastz allows a transition $(A \leftrightarrow G, C \leftrightarrow T)$ at any one of the 12 match positions. This is the first example, albeit empirical, of differentiating between transitions and transversions in alignments. Later, Noe and Kucherov [30] used seed patterns of 0,1 and \# (transition) symbols coupled with a Bernoulli alignment model for non-coding regions and a hidden Markov model for coding regions to search for optimal spaced seeds of weights 9 to 11, which they implemented in their program YASS [41]. In different types of applications, Csuros and $\mathrm{Ma}$ [42] developed an algorithm to reduce the memory usage for multiple spaced seeds, and Flannick and Batzoglou [43] used multiple spaced seeds in an algorithm to improve local alignment sensitivity. Lastly, multiple spaced seeds with two mismatch positions were implemented in the tool zoom [44] for fast, high-throughput mapping of short sequencing reads to a target genome.

2.5. Spaced Seeds for cDNA-to-Genome Alignment. Early work in designing theoretically sensitive seeds has focused on seeds for aligning arbitrary genomic sequences $[21-23,27,31,35,36]$, due primarily to the simplicity of the alignment model. As genomic sequences can be well approximated by a random model for most practical problems, a Bernoulli model is suitable in this case. A smaller number of studies addressed optimal seeds for cDNA-to-genome alignments, usually as a particular case of the general problem $[21,27,28]$.

Unlike genomic sequences, in which less than $2 \%$ of nucleotides typically represent coding material, cDNA sequences consist largely of protein coding regions, and thus cDNA-to-genome alignments are expected to have more embedded sequence compositional structure than alignments of random DNA. This includes effects of transition-transversion biases [45], higher order statistical dependencies between positions [2], and periodicities due to the codon organization of sequences. These biases translate into dependencies in the match patterns and, when incorporated into the alignment model, are likely to lead to better seeds. 
In the following section we describe our recent efforts with developing better models for cDNA-to-genome alignment starting from the observations above, and use them to illustrate the seed design framework.

3. Designing Sensitive and Specific Spaced Seeds. We illustrate the mathematical framework of seed design by focusing on the case of cDNA-to-genome alignment. We review the basic $\{0,1\}$ match-mismatch framework [22], then present an extension that is better suited for this particular problem. In this context, using efficient methods for estimating sensitivity and specificity we evaluate a large number of seeds for a variety of seed weights, to assess their sensitivity-specificity tradeoffs and ultimately design accurate seeds for practical applications.

\subsection{Designing Sensitive Spaced Seeds.}

3.1.1. The Basic $\{\mathbf{0}, \mathbf{1}\}$ Framework. As we have mentioned earlier, the original theoretical framework for assessing seed sensitivity has two components: a seed model and an alignment model. A seed is a string of 0 s and 1s that specifies a match pattern between the sequences, where 1s represents match positions and 0s represent wildcards. For instance, the WABA seed 11011011011011011 has the wildcard positions 3, 6, 9, 12 and 15. Conventionally, seeds must start with a 1 position. For simplicity, a fixed span has been used in most cases (e.g., $k=22$ ) [21,24,27] assuming terminating positions to be 0 s wherever necessary.

An alignment is represented as a string of 0s (mismatches) and 1s (matches) generated from a model $\mathcal{M}$, typically a Bernoulli or a Markov model. A seed $S=$ $s_{1} \ldots s_{k}, s_{1}=1$, is said to detect the alignment $\mathcal{A}=a_{1} \ldots a_{L} \in\{0,1\}^{L}$ if there is a match for the pattern in the alignment string such that all 1 positions in the seed pattern map to 1 positions in the alignment, i.e. there exists an index $i$ between 1 and $L-k+1$ such that $a_{i+l-1}=1$ for all $l$ with $s_{l}=1$ (Figure 1 ). In that case, the seed is said to occur in the alignment $a$ at position $i$. Given a seed, its theoretical sensitivity is defined as the probability that it will detect a random alignment of length $L$ generated from the model $\mathcal{M}[21,23]$, or equivalently:

$$
\operatorname{Sn}(S)=P\left(\left\{\mathcal{A} \in\{0,1\}^{L} \mid S \operatorname{detects} \mathcal{A}\right\}\right) .
$$

Traditionally, the alignment length $L$ has been set to 64 , which is the average length of a gap-free alignment in human-mouse comparisons. For a given seed, its sensitivity can be computed exactly using dynamic programming [23, 27,33], and optimal or near-optimal seeds can be obtained with the exact or heuristic methods reviewed in the previous section.

3.1.2. The Extended Codon-Sensitive $\{0,1, x\}$ Framework. Alignments of coding sequences have characteristics that differentiate them from genomic sequence 


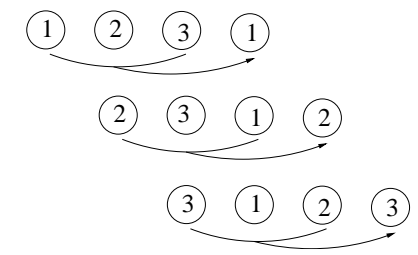

FIG. 2. Position dependencies for an order 3 inhomogeneous, 3-periodic Markov model.

alignments, such as transition-transversion biases, higher order dependencies between positions and periodicities due to the codon structure, and therefore require more complex models. To account for the first of these characteristics, in previous work we proposed a framework that differentiated between transitions and transversions, by using an additional alphabet symbol $x$ [24]. In the seed pattern, $x$ marks a position that allows transitions $(\mathrm{A} \leftrightarrow \mathrm{G}, \mathrm{C} \leftrightarrow \mathrm{T})$ but not transversions, while in the alignment model it simply represents a transition (Figure 1). Furthermore, to reflect the periodicity and higher order dependencies in the sequences of coding regions, the alignment was modeled with a 3-periodic inhomogeneous Markov model consisting of 3 submodel components, one for each position in the codon (Figure 2). The individual submodels are Markov models of the same order $m(m=0-5)$. The definitions and methods can be extended from the $\{0,1\}$ case in a straightforward way. The weight of the new symbol $x$ is 0.5 . We denote $\left(n_{1}, n_{0}, n_{x}\right)$ the class of seed patterns with $n_{1}, n_{0}$ and $n_{x}$ symbols of 1,0 and $x$, respectively, $n_{1}+n_{0}+n_{x}=k$. Note that for a given weight $W$, there may be multiple $\left(n_{1}, n_{0}, n_{x}\right)$ combinations with $n_{1}+0.5 \cdot n_{x}=W$. For instance, for the weight $W=10$ and span $k=22$ the following combinations are possible: $(10,12,0),(9,11,2), \ldots,(0,2,20)$.

Theoretical Sensitivity Evaluation. To estimate seed sensitivity, we adopted and extended the method in [21] and the associated mandala software to allow transition symbols. Briefly, the method builds a trie of all the alignment words that represent seed matches, then converts the trie into a deterministic finite automaton (DFA) that recognizes all and only those alignment strings containing a seed match, using the Aho-Corasick algorithm [32]. The sensitivity of the seed is then computed recursively from the automaton as the probability of all alignment strings of length $L=64$ that are recognized by the automaton. The detailed implementation for the $\{0,1, x\}$ case can be found in [24].

Empirical Evaluation. We complemented our theoretical studies with empirical evaluations, both to identify a suitable alignment model and to validate our predictions. Specifically, empirical tests were performed to test the ability of a seed to 
accurately match coding exons between two species, for instance when searching for chicken exons against the human genome [24]. Empirical sensitivity was calculated as the fraction of human exons in the reference set that are detected by the seed: $S n^{e}=T P /(T P+F N)[46]$. Here, TP is the number of true positives, defined as orthologous exon pairs detected by the seed, and FN represents the numbers of false negatives, or missed pairs.

Comparisons between the empirical and the predicted behavior of seeds revealed that higher order Markov models $(m=3-5)$ are more accurate descriptors of the empirical data and lead to better seeds than those produced by earlier lower-order models. Additionally, changing a 0 into a transition symbol in a $\{0,1\}$ seed roughly doubles the specificity for only a small decrease in sensitivity, and thus offers a better sensitivity-specificity tradeoff compared to $\{0,1\}$ seeds in practice $[24,30]$. Based on these analyses, an order 3 inhomogeneous 3 -periodic Markov model of alignment was chosen for our comprehensive seed evaluation in section 3.3.

3.2. Designing Specific Spaced Seeds. While sensitivity has been at the forefront of seed design efforts, specificity has received considerably less attention. Decreasing the seed weight generally increases sensitivity, but at a loss in specificity. Understanding the tradeoffs between these two properties is essential to designing accurate seeds that can work efficiently in practical alignment applications. We present the theoretical framework and methods we proposed in [24] for evaluating seed specificity in the case of $\{0,1, x\}$ spaced seeds for cDNA-to-genome alignment. Similar methods need to be developed for other types of models and seeds.

3.2.1. Evaluating Seed Specificity. We begin by presenting the intuition behind our definition of specificity. When mapping cDNA sequences to a target genome, consecutive $k$-mers in the cDNA query are searched against the genome sequence. The search will find at most one true positive match, namely its ortholog on the genome, and a variable number of false positives, which add to both processing time and program complexity. Thus, intuitively, we define the specificity of a seed as the inverse of the expected number of matches in the genome for a generic $k$-mer via the particular seed, $\mathcal{H}(S)$ :

$$
S p(S)=\frac{1}{\mathcal{H}(S)}=\frac{1}{E_{S}[H]}
$$

Here, $H(w)$ is a random variable representing the expected number of (overlapping) matches of a $k$-mer $w$ in the genome sequence via seed $S$. Assuming probabilistic models for both the gene and the genomic sequences, the above term can be written as: 


$$
E_{S}[H]=\sum_{w \in\{A, C, G, T\}^{k}} P_{c}(w) H(w)=\sum_{w \in\{A, C, G, T\}^{k}} P_{c}(w) E\left[N_{S}(w)\right]
$$

where $P_{c}(w)$ is the probability of $w$ in the coding sequences, and $N_{S}(w)$ is a random variable denoting the number of matches of $w$ in the genome via seed $S$. For a continuous seed, this is the number of occurrences of $w$ in the genome. For a spaced seed containing wildcard positions, it is the number of occurrences of all words $w^{\prime}$ that are compatible with $w$ via seed $S$. For instance, for the word $A A G C T$ and the seed $S=1 x 111$, the set of compatible words is $\{A A G C T, A G G C T\}$.

In [24] we derived a closed formula and recurrences to efficiently calculate seed specificity for the case of Bernoulli and Markov models of gene sequences and for a Bernoulli or order 1 Markov model of the genome.

Theoretical Evaluation. The definition and recurrences mentioned above allowed us to calculate specificity values for a large number of seeds exactly and efficiently. Speciesspecific mRNA sequences were downloaded from the NCBI RefSeq repository [9] and used to train Bernoulli and Markov models of coding sequences. Similarly, nucleotide and di-nucleotide frequencies obtained from the human genome version HG17 were used to train the two genome models.

Empirical Evaluation. As before, empirical testing was performed to calibrate and validate the theoretical models. Empirical specificity was calculated as the inverse of the average number of seed matches in the human genome for a generic $k$-mer in the query: $S p^{e}=1 /((T P+F P) / \# \mathrm{k}$-mers $)$. This definition mirrors the theoretical formulation described earlier.

Comparisons of empirical and predicted seed behavior allowed us to determine the most accurate model for use in our comprehensive evaluation. As we found earlier for sensitivity, higher-order Markov models of coding sequences provided a highly accurate approximation of the real specificity values, while the Markov genome model best captured the actual amount of specificity change as the weight of the seed decreased. Therefore, an order 1 Markov genome model and order 2 Markov coding sequence models were chosen for further evaluations.

3.3. Comprehensive Seed Evaluation. The models and methods developed above allowed us to evaluate a large number of seeds in a short amount of time, to assess their tradeoffs between sensitivity and specificity. We analyzed seeds in seven weight groups $(W=10 \ldots 16)$ and for all feasible $\left(n_{1}, n_{0}, n_{x}\right)$ combinations. For each combination, the hill-climbing heuristic described in section 2 was used to estimate the maximum and minimum sensitivity and specificity. The results are plotted linearly 
for sensitivity, and on a log-scale for specificity, for the particular case of humanchicken comparison (Figure 3). Similar plots were obtained for other species (data not shown).
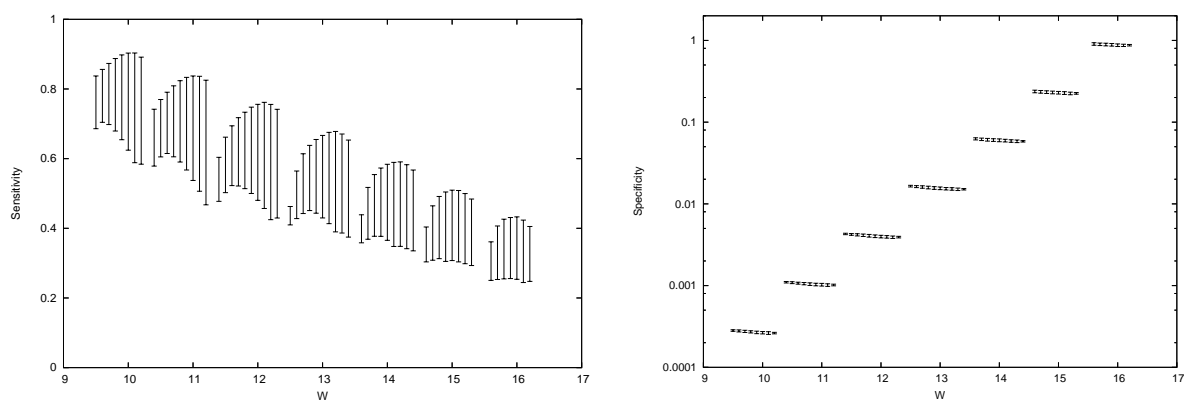

FIG. 3. Sensitivity (left, linear-scale) and specificity (right, log-scale) ranges for seeds of weight $W=10 \ldots 16$, using an order 3 Markov model alignment representation for sensitivity evaluation, and using an order 1 genome Markov model and an order 2 Markov model for coding sequences for specificity. For each weight $W,\left(n_{1}, n_{0}, n_{x}\right)$ combinations are shown right-to-left starting with $n_{x}=0$ and subsequently increasing $n_{x}$. Reproduced from [24] with permission from Mary Ann Liebert, Inc.

As the plots illustrate, sensitivity varies widely among seeds within each weight group and each $\left(n_{1}, n_{0}, n_{x}\right)$ combination. Although the combinations have different ranges of variation and maximum sensitivity, the pattern of variation is consistent among the different weight groups. Furthermore, for the chicken-human comparison we used as example, the highest sensitivity was achieved for seeds with a small (2-4) number of transition symbols. This number is comparison-specific and varies with the transition-transversion ratio [25]. For instance, the human-mouse comparison, which has a higher transition-transversion ratio $\left(\kappa_{m}=1.73>\kappa_{c}=1.17\right)$, shows an increased affinity for transition symbols (6-8). Furthermore, this number reflects the gain in sensitivity that can be obtained by incorporating transitions compared to $\{0,1\}$ seeds.

In contrast, specificity is almost constant within each weight group, and shows a discrete range of steps. As the weight of the seed increases, specificity grows by a factor of roughly 3.6 with each additional unit. These observations suggest the following strategy for selecting seeds for practical applications: first determine the seed weight based on an acceptable sensitivity-specificity tradeoff, then select the most sensitive seed in that weight group.

4. Other Problems in Seed Design. The framework and methods described in the previous sections were designed to optimize seeds for one comparison at a time. With the improvement in sequencing technologies, the number of sequenced organisms has grown significantly in recent years, with more genomes and other sequence data to become available in the near future. Finding optimal seeds for each 
pairwise comparison quickly becomes prohibitive, both because of the computational costs involved and because of the difficulty that it poses to alignment program developers and users. For practical reasons, therefore, it becomes desirable to identify a small number of seeds that would perform well for a wide range of comparisons. Several studies [31,35,36] determined and analyzed best seeds for various sequence identity levels $p$ under a Bernoulli model of alignment, in a greatly simplified model of evolutionary distance. This simple $p$-level representation of evolutionary sequence divergence is likely inadequate for coding sequences, which are under a more diverse set of evolutionary pressures. In this section we present two recent approaches: i) finding universal (good) seeds, or seeds that work well across different classes of comparisons [25], and ii) clustering comparisons into seed-equivalent groups and determining best seeds for each group [26].

4.1. Determining Universal Seeds. We sought to observe the behavior of seeds, and in particular good seeds, across different classes of comparisons, with the goal to characterize and ultimately determine universal seeds. We focused on four comparisons (human-mouse, human-dog, human-chicken, and human-zebrafish), which coarsely sample the range of vertebrate evolutionary distances. For simplicity, we will refer to each comparison by the name of the second organism (DOG, MUS, CHK, ZFS). Using the extended framework of the previous section, we evaluated seeds exhaustively for several weights, and analyzed the distributions of seed sensitivities between the models.

Statistical regression analyses applied to seed sensitivities between pairs of comparisons have shown that there is good correlation among the models, and remarkably strong correlation between some pairs of models (Figure 4). Indeed, to determine seed trends we measured the $95 \%$ confidence interval when projecting the sensitivity value of a seed from the reference model to the compared model via the regression function. This margin of error was very small $(<0.01)$ when projecting from a more distant comparison to a closer one, and bi-directionally between the pairs CHK-ZFS and MUS-DOG, indicating that with high confidence (95\%) seed sensitivities can be accurately projected between these models. In particular, high-scoring seeds in the reference model are expected to lie at the top of the sensitivity range in the compared model for these cases.

We further measured how closely the projection of the optimal seed in the reference model $\left(x_{\max }\right)$ estimates the optimal (real) seed in the compared model $\left(y_{\max }\right)$, using the statistical lower bound of the confidence interval for $x_{\max }$ as a conservative estimate and comparing it with $y_{\max }$. These ratios are shown as $T(x, y)$ in Table 1. When the optimal seed in a more distant model is projected onto a closer model its ratio is consistently greater than 0.98 , and thus it closely approximates the optimal seed in that model. 

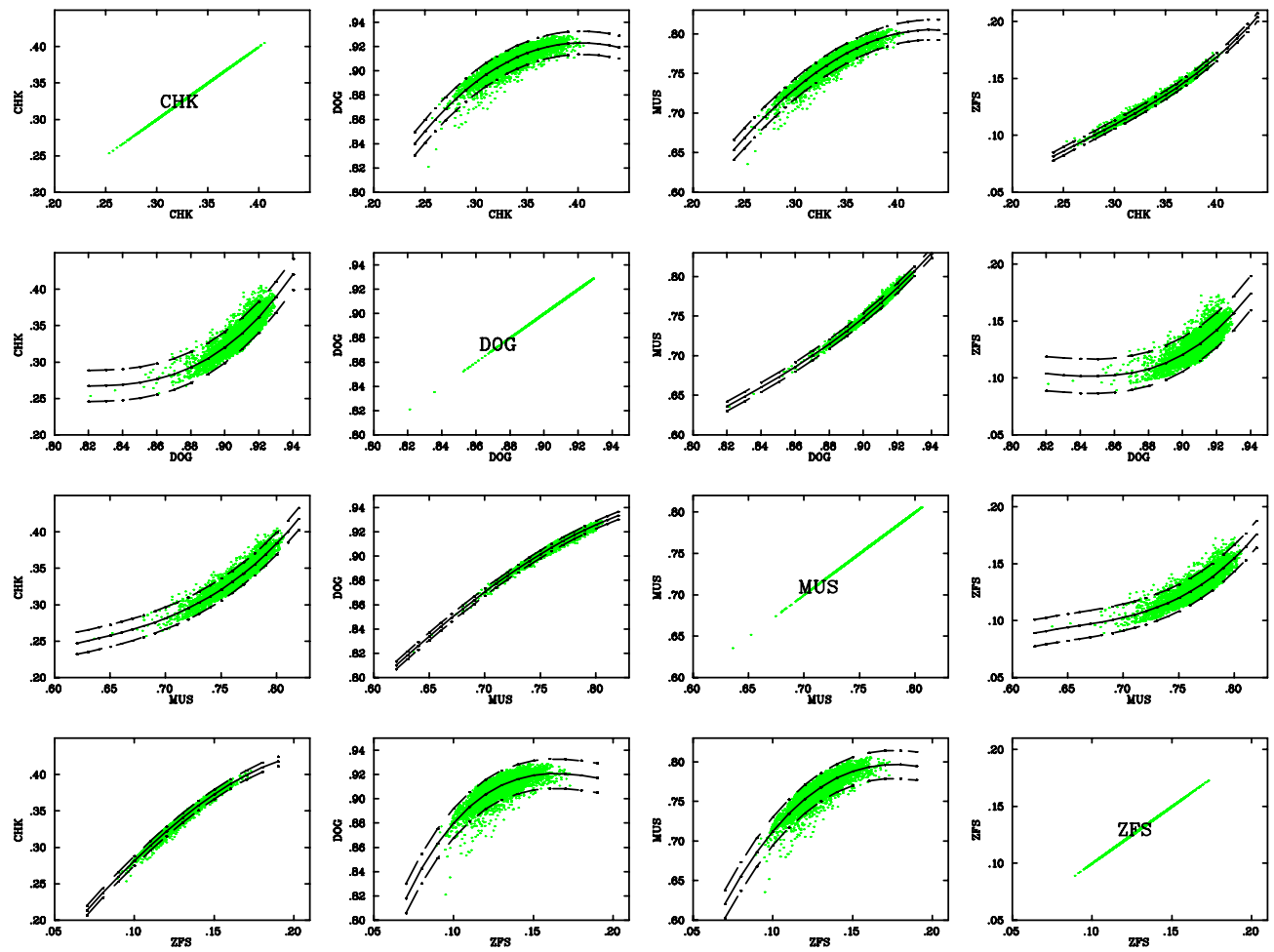

FIG. 4. Scatterplots of seed sensitivity values for pairs of comparisons among (DOG, MUS, CHK, ZFS). The non-linear regression (solid) and the 95\% confidence interval (dashed) curves are shown.

These findings, validated by empirical evaluations, suggest that simply selecting top scoring seeds in the most distant model, in our case ZFS, with high probability will lead to good seeds for all other models, and therefore provide a simple strategy for determining universal good seeds.

There is yet another observation critical for designing seeds for multiple comparisons that this analysis revealed: similar comparisons render similar behavior of seeds, and in particular best seeds. We call such pairs of comparisons seed-equivalent. In our case, (ZFS, CHK), and (DOG,MUS) are sufficiently close to be seed-equivalent. In the following section we exploit this finding in determining conditions for seed-equivalence and employ them to cluster comparisons into groups that each can be served by the same optimal seed.

4.2. Determining Seeds for Seed-Equivalent Comparisons. We observed the existence of seed-equivalent models when comparing seed behavior between comparisons with statistical regression methods, using exhaustive calculations of seed sensitivities. One outstanding question is how to determine whether two models are 
TABLE 1

Comparison of seed sensitivity distributions between models. $y_{\min , \max }$ are the minimum and maximum sensitivity in the compared model; $x_{\max }$ is the maximum sensitivity in the reference model; $t_{\alpha / 2} \sigma_{y}$ is half the length of the $95 \%$ prediction interval $\left(t_{\alpha / 2}=1.96\right.$ when $\left.\alpha=0.05\right)$; $T(x, y)=\left(\left(a+b x_{\max }+c x_{\max }^{2}+d x_{\max }^{3}\right)-t_{\alpha / 2} \sigma_{y}\right) / y_{\max }$, where $a, b, c$ and $d$ are the coefficients of the regression curve, and $\sigma_{y}$ is the estimated regression standard error of prediction for a given $x$ value. Because the number of values is large, $\sigma_{y} \simeq \sigma$ for all $y$. Reproduced from [25] with permission from BioMed Central Ltd.

\begin{tabular}{|l|c|c|c|c|c|}
\hline & $x_{\max }$ & $y_{\max }$ & $y_{\min }$ & $t_{\alpha / 2} \sigma_{y}$ & $T(x, y)$ \\
\hline \multicolumn{6}{|c|}{ W=12, population size $=352716}$. \\
\hline CHK-DOG & 0.742 & 0.992 & 0.936 & 0.002 & 0.996 \\
DOG-CHK & 0.992 & 0.742 & 0.429 & 0.028 & 0.947 \\
CHK-MUS & 0.742 & 0.964 & 0.827 & 0.005 & 0.991 \\
MUS-CHK & 0.964 & 0.742 & 0.429 & 0.017 & 0.978 \\
CHK-ZFS & 0.742 & 0.476 & 0.196 & 0.006 & 0.995 \\
ZFS-CHK & 0.476 & 0.742 & 0.429 & 0.006 & 0.984 \\
DOG-MUS & 0.992 & 0.964 & 0.827 & 0.004 & 0.996 \\
MUS-DOG & 0.964 & 0.992 & 0.936 & 0.001 & 0.999 \\
DOG-ZFS & 0.992 & 0.476 & 0.196 & 0.033 & 0.866 \\
ZFS-DOG & 0.476 & 0.992 & 0.936 & 0.003 & 0.995 \\
MUS-ZFS & 0.964 & 0.476 & 0.196 & 0.023 & 0.934 \\
ZFS-MUS & 0.476 & 0.964 & 0.827 & 0.006 & 0.988 \\
\hline
\end{tabular}

sufficiently close to be seed-equivalent without the expensive calculation of seed sensitivities. Once a suitable measure for model similarity is determined, it can be used to cluster comparisons into seed-equivalent groups and then design seeds for each group. We ask the following questions: i) How do we measure the closeness of comparisons? ii) How do we group comparisons? iii) How do we validate the groups?

We investigate the relationship between alignment Markov models using a conventional distance measure between their probability distributions. The Kullback-Leibler Divergence (KLD) [47] can be applied on the space of alignment words $\mathcal{X}=\{0,1, x\}^{64}$ to produce a distance between the two models:

$$
K L D(P, Q)=\sum_{w \in \mathcal{X}} p(w) \log \frac{p(w)}{q(w)}
$$

$K L D(P, Q)$ represents the relative entropy of $P$ over $Q$, or the information gain about $\mathcal{X}$ when $P$ is used instead of $Q$. The KLD measure is non-symmetrical, i.e. $K L D(P, Q) \neq K L D(Q, P)$, and therefore can capture unidirectional relationships. In the case of the four comparisons above (DOG, MUS, CHK and ZFS), the more distant comparisons contain consistently more information than the closer ones [25]. A metric distance, which is symmetrical, can then be defined as $\frac{1}{2}(K L D(P, Q)+K L D(Q, P))$. 


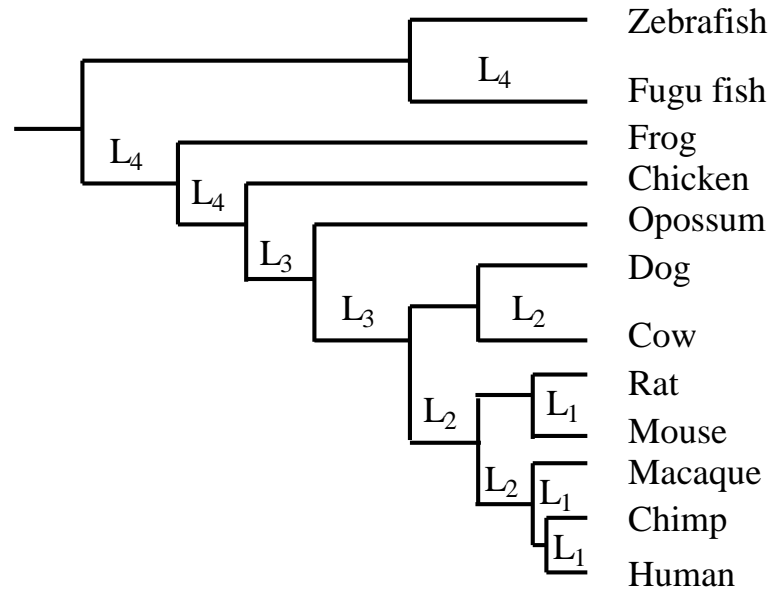

FIG. 5. Phylogenetic tree of 12 species.

To validate the metric, we applied it to a larger set of species, including human, chimp, macaque, mouse, rat, cow, dog, opossum, chicken, frog, fugu, and zebrafish. The comparisons are not limited to those between human and other species, but rather extend to all 66 pairwise comparisons of the 12 species, and thus offer a wide diversity of evolutionary distances and mutation patterns. KLD distances between Markov models were calculated and used to generate a distance profile for each comparison. A profile is the vector of KLD distances of one comparison with all comparisons. Profiles were then clustered using hierarchical clustering with the Pearson correlation coefficient to create groups of similar comparisons.

Remarkably, the 66 comparisons fall into four clusters [26] closely corresponding to the different depths of the phylogenetic tree of the species (Figure 5): cluster $L_{1}$ contains comparisons between evolutionarily very close species (5-12 Million Years divergence) at or close to the leaf-level in the phylogenetic tree of the species; cluster $L_{2}$ represents comparisons at the intermediate (90 - 110 Million Years divergence), between primates and the rodent, artyodactil or carnivore species; cluster $L_{3}$ shows comparisons between relatively diverged species (160-310 Million Years divergence), such as between chicken or opossum and the rest of the mammals; lastly, cluster $L_{4}$ represents some of the most distant comparisons among vertebrates (>350 Million Years divergence), between frog or fishes and the rest of the clades, at the deepest levels in the phylogenetic tree.

We optimize seeds for each cluster and for each comparison in the clusters, using the framework described earlier, and validate them by comparing their performance within their group and in the other groups. Indeed, seeds optimized for one cluster performed best on their own cluster and on the individual comparisons within that cluster. Based on this large scale clustering and validation analysis, it can be 
concluded that our method of clustering models based on their KLD distance profile followed by optimizing seeds for each group can be effectively and reliably used to determine a small number of seeds with wide applicability among a variety of comparisons.

These findings have important implications for developing robust and user-friendly alignment tools [48], by providing program developers with a small and easily tunable set of parameters, and providing a unified and easy to use interface to the users, who can now seamlessly use the tool without regard to the species compared.

4.3. Applying Seeds to Gene Detection. Gene annotation by mapping foreign cDNAs to genomic sequence will fail when no copies of a gene are available. However, sufficient information is present in the genomic alignments to allow inferring gene regions. Indeed, gene regions will exhibit higher sequence similarity compared to non-coding DNA, as natural selection lowers the rates at which mutations are incorporated in gene regions. Moreover, they show higher-level dependencies between positions and periodicities due to the codon organization of genes, all of which differentiate them from other DNA sequences. Seeds optimized for cDNA-to-genome comparisons capture the characteristics of gene regions in sequence alignments in a natural way, and therefore may be apt to locate genes for which no other information is available.

We have started to explore the use of seeds to differentiate between gene and non-gene regions by exploiting the differences in their match patterns among the three reading frames. Indeed, some seeds appear to match differentially among the three reading frames when applied to coding regions, having a significantly higher or lower match probability in one of the frames compared to the others [24], whereas they show no bias in non-coding regions. To assess empirically the ability of codonsensitive seeds to distinguish among the three frames, we applied seeds derived in our previous studies [25] to the coding region alignments of 17,670 orthologous human and mouse mRNA sequences obtained from the project HomoloGene [49], counting the number of seed matches within the alignment strings for each frame (Figure 6). Since in eukaryotic species coding regions appear as short exons interspersed with introns, we performed a similar experiment on sets of short coding regions of fixed length (51 bp, $102 \mathrm{bp}, 150 \mathrm{bp}$ and $201 \mathrm{bp}$ ) cut from the alignments of full-length sequences. These preliminary analyses reveal significant frame differences when applying specific transition spaced seeds to all three frames of human-mouse pairwise alignments of gene sequences. Although the sensitivity decreases slightly with the length of the target regions, this simple seed match counting method achieves $92 \%$ sensitivity on the $150 \mathrm{bp}$ data set, corresponding to the average length of coding exons. Thus, spaced seeds appear to be a promising instrument for detecting genes in genomic sequence alignments. The theoretical foundations for selecting sensitive 

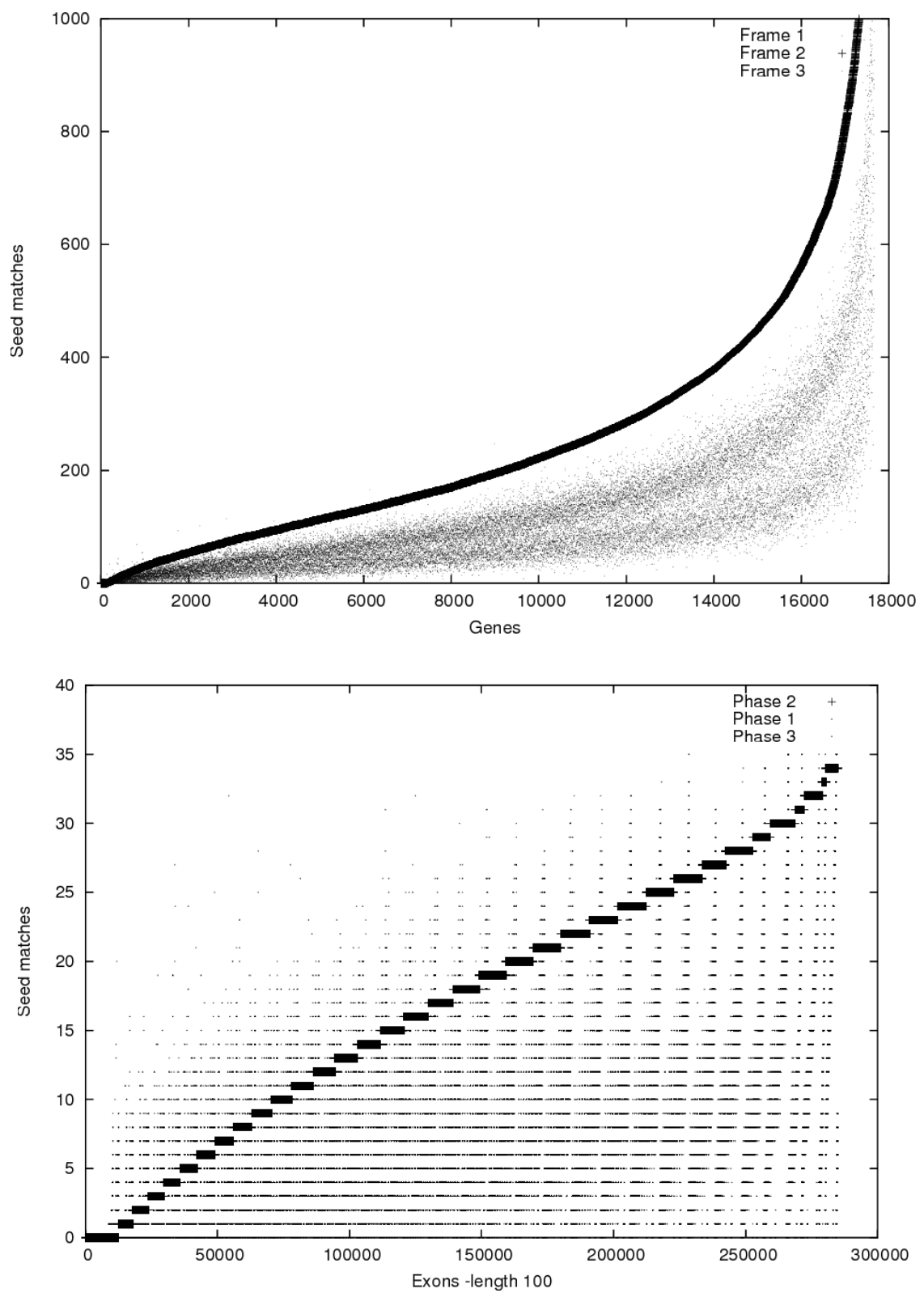

FIG. 6. Gene detection with seeds: differential frame analysis of alignments of full-length (top) and exon-size (bottom) coding regions. The numbers of seed matches in all three frames are plotted for 17,670 alignments of human and mouse gene sequences, sorted increasingly by frame 2. The test seed matches preferentially in frame 2 (thick line).

seeds with a high discriminative power, and the potential of different classes of seeds for practical applications, need to be further explored and developed. 
5. Conclusions. We reviewed recent developments in spaced seed design for cross-species cDNA-to-genome alignment, starting from the original framework and continuing with extensions to address both traditional and novel problems in seed design. Compared to genomic sequences, which are roughly randomly distributed, gene sequences have significantly more sequence compositional structure, such as periodicities due to codon organization, higher-order position dependencies and characteristic transition-transversion biases. By incorporating these features into a new framework, which allows transition wildcards within seeds, we were able to find more accurate, higher-order models that produced more sensitive seeds.

Designing good seeds for accurate alignment programs must balance sensitivity and specificity. We present a first theoretical framework we developed earlier for estimating the specificity of a seed, based on its expected number of matches in the genome sequence, and use it to analyze the sensitivity and specificity of a large number of seeds in parallel. These analyses showed that while sensitivity varies widely among seeds with the same weight, specificity is relatively constant. In particular, they clearly indicate that spaced seeds with transitions designed by these models offer better sensitivity-specificity tradeoffs compared to traditional $\{0,1\}$ seeds. Furthermore, the interplay between sensitivity and specificity suggests a clear strategy to determine the best seeds in practice, by first choosing the desired level of specificity and sensitivity tradeoff by selecting the seed weight, and then selecting the most sensitive seed within that weight group. This comprehensive and systematic seed evaluation provides the blueprint for selecting accurate, i.e. both sensitive and specific, seeds for a wide variety of practical applications.

As the number and variety of sequenced species increases, for practical reasons it becomes desirable to determine a small number of seeds that are applicable to a wide range of comparisons. This new consideration brings a shift from the single to the multi-comparison paradigm in seed design. We propose two solutions, namely determining universal seeds that work well for classes of comparisons across evolutionary distances and, at a higher resolution, designing seeds for groups of seed-equivalent comparisons, clustered based on model similarity. These findings have important implications for developing robust programs that are easy to use, with a relatively small set of parameters that can be accurately tuned and easily understood by the users. Lastly, we proposed that spaced seeds may be used as 'machine-learning' models in other areas of computational biology, such as gene finding.

Future work will be needed to extend the methods presented here, in particular mapping the sensitivity-specificity tradeoffs, to other categories of seeds, to improve the efficiency of seed design for single and multiple comparisons, to design seeds and new seed models to support the alignment of sequences produced by new sequencing technologies, to develop more efficient ways to incorporate seeds into alignment tools, and to explore the potential of seeds in other application areas. 
Acknowledgments. This work was supported in part by a Sloan Research Fellowship to Liliana Florea. The comprehensive seed evaluation was performed on the "Herd" Scientific Computing Cluster at The George Washington University.

\section{REFERENCES}

[1] National Human Genome Research Institute, Listing of Genome Sequencing Proposals. [http://www.genome.gov/10002154].

[2] C. Burge and S. Karlin. Prediction of complete gene structures in human genomic DNA. J Mol Biol. 268 (1997), pp. 78-94.

[3] D. Kulp, D. Hussler, M. Reese, and F. Eeckman. A generalized hidden Markov model for the recognition of human genes in DNA. Proc Int Conf Intell Syst Mol Biol. 4(1996), pp. $134-142$.

[4] A. Lukashin and M. Borodovsky. GeneMark.hmm: New solutions for gene finding. Nucleic Acids Res. 26(1998), pp. 1107-1115.

[5] A. Salamov and V. Solovyev. Ab initio gene finding in Drosophila genomic DNA. Genome Res. 10:4(2000), pp. 516-522.

[6] L. Florea, V. Di Francesco, J. Miller, R. Turner, C. Mobarry, A. Yao, M. Harris, B. Walenz, i. Dew, G. Merkulov, R. Charlab, Z. Deng, S. Istrail, P. Li, and G. Sutton. Gene and alternative splicing annotation with AIR. Genome Res. 15(2005), pp. $54-66$.

[7] Y. Okazaki, M. Furuno, T. Kasukawa, J. Adachi, H. Bono, S. Kondo, I. Nikaido, N. Osato, R. Saito, and H. Suzuki et Al. Analysis of the mouse transcriptome based on functional annotation of 60,770 full-length cDNAs. Nature, 420(2002), pp. 563-573.

[8] MGC Project Team. The status, quality, and expansion of the NIH full-length cDNA project: the Mammalian Gene Collection (MGC). Genome Res. 14(2004), pp. 2121-2127.

[9] K. Pruitt, T. Tatusova, and D. Maglott. NCBI Reference Sequence (RefSeq): a curated non-redundant sequence database of genomes, transcripts and proteins. Nucleic Acids Res. 35(2007), pp. D61-D65.

[10] L. Florea, G. Hartzell, Z. Zhang, G. Rubin, W. Miller. A computer program for aligning a cDNA sequence with a genomic DNA sequence. Genome Res. 8:9(1998), pp. 967-974.

[11] S.J. Wheelan, D.M. Church and J.M. Ostell. Spidey: a tool for mRNA to genomic alignments. Genome Res. 11:11(2001), pp. 1952-1957.

[12] W.J. Kent. BLAT - the BLAST-like alignment tool. Genome Res. 12(2002), pp. 656-664.

[13] T.D. Wu AND C.K. WATANABE. GMAP: a genomic mapping and alignment program for $m R N A$ and EST sequences. Bioinformatics, 21(2005), pp. 1859-1875.

[14] B.T.K. Lee, T.W. TAn, And S. Ranganathan. MGAlignIt: a web service for the alignment of $m R N A / E S T$ and genomic sequences. Nucl. Acids Res. 31:13(2003), pp. 3533-3536.

[15] M. Zhang AND W. Gish. Improved spliced alignment from an information theoretic approach. Bioinformatics. 22:1(2006), pp. 13-20.

[16] J. Usuka, W. Zhu, and V. Brendel. Optimal spliced alignment of homologous cDNA to a genomic DNA template. Bioinformatics. 16:3(2000), pp. 203-211.

[17] R. Мотт. EST_GENOME: a program to align spliced DNA sequences to unspliced genomic DNA. Comput. Appl. Biosci. 13:4(1997), pp. 477-478.

[18] S. Altschul, W. Gish, W. Miller, E. Myers, D. Lipman. Basic local alignment search tool. J Mol Biol. 215:3(1990), pp. 403-410.

[19] S. Altschul, T. Madden, A. Schaffer, J. Zhang, Z. Zhang, W. Miller, D. Lipman. Gapped BLAST and PSI-BLAST: A new generation of protein database search programs. Nucleic Acids Res. 25:17(1997), pp. 3389-3402. 
[20] W. Kent And A. Zahler. Conservation, regulation, synteny, and introns in a large-scale C. briggsae-C. elegans genomic alignment. Genome Res. 10:8(2000), pp. 1115-1125.

[21] J. Buhler, U. KeICH U, AND Y. Sun. Designing seeds for similarity search in genomic DNA. In Proceedings of the Seventh Annual International Conference on Computational Molecular Biology (RECOMB 2003). 7(2003), pp. 67-75.

[22] B. Ma, J. Tromp, And M. Li. PatternHunter: faster and more sensitive homology search. Bioinformatics. 18:3(2002), pp. 440-445.

[23] U. Keich, M. Li, B. Ma, And J. Tromp. On spaced seeds for similarity search. Discrete Appl Math. 138:3(2004), pp. 253-263.

[24] L. Zhou And L. Florea. Designing sensitive and specific spaced seeds for cross-species mRNAto-genome alignment. J Comput Biol. 14:2(2007), pp. 113-130.

[25] L. Zhou, J. Stanton, And L. Florea. Universal seeds for cDNA-to-genome comparison, BMC Bioinformatics, 9:36(2008).

[26] L. Zhou, I. Mihai, and L. Florea. Effective cluster-based seed design for cross-species sequence comparison. Bioinformatics, 24:24(2008), pp. 2926-2927.

[27] J. Buhler, U. Keich, Y. Sun. Designing seeds for similarity search in genomic DNA. J Comput Syst Sci. 70:3(2005), pp. 342-363.

[28] B. Brejová, D. Brown, AND T. Vinar. Optimal spaced seeds for homologous coding regions. J Bioinform Comp Biol. 1:4(2004), pp. 595-610.

[29] S. Schwartz, W. Kent, A. Smit, Z. Zhang, R. Baertsch, R. Hardison, D. Haussler, AND W. MiLLER. Human-mouse alignments with BLASTZ. Genome Res. 13(2003), pp. $103-107$.

[30] L. Nó́ and G. Kucherov. Improved hit criteria for DNA local alignment. BMC Bioinformatics. 5:149(2004),

[31] K. Chor And L. Zhang. Sensitivity analysis and efficient method for identifying optimal spaced seeds. J Comp Syst Sci. 68(2004), pp. 22-40.

[32] A. Aho AND M. Corasick. Efficient string matching: an aid to bibliographic search. Comm ACM. 18:6(1975), pp. 333-340.

[33] G. Kucherov, L. NoÉ, And M. Roytberg. A unifying framework for seed sensitivity and its application to subset seeds. J Bioinform Comp Biol. 4:2(2006), pp. 553-569.

[34] G. Kucherov, L. NoÉ, And M. Roytberg. Subset Seed Automaton, Proceedings of the 12th International Conference on Implementation and Application of Automata (CIAA 2007), Prague, Czech Republic, July 16-18, 2007. LNCS, 4783(2007), pp. 180-191. Holub J and Zdarek J, Eds.

[35] K. Choi, F. Zeng, and L. Zhang. Good spaced seeds for homology search. Bioinformatics. 20:7(2004), pp. 1053-1059.

[36] F. Preparata, L. Zhang, and K.P. Choi. Quick, practical selection of effective seeds for homology search. J Comput Biol. 12:9(2005), pp. 1137-1152.

[37] L. Ilie AND S. ILIE. Multiple spaced seeds for homology search, Bioinformatics, 23:22(2007), pp. 2969-2977.

[38] M. Li, B. Ma, D. Kisman, And J. Tromp. PatternHunter II: highly sensitive and fast homology search. J Bioinf Comp Biol. 2(2002), pp. 411-439.

[39] Y. Sun And J. Buhler. Designing multiple simultaneous seeds for DNA similarity search. J Comput Biol. 12:6(2005), pp. 847-861.

[40] B. Brejová, D. Brown, And T. Vinar. Vector seeds: an extension to spaced seeds. J Comput Syst Sci. 70:3(2005), pp. 364-380.

[41] L. NoÉ And G. Kucherov. YASS: enhancing the sensitivity of DNA similarity search. Nucleic Acid Res. 33(2005), pp. W540-W543.

[42] M. Csuros And B. Ma. Rapid homology search with two-stage extension and daughter seeds. In: COCOON 2005 - Eleventh International Computing and Combinatorics Conference. 
Springer LNCS 3595 (2005), pp. 104-114.

[43] J. Flannick And S. Batzoglou. Using multiple alignments to improve seeded local alignment algorithms. Nucleic Acids Res. 33:14(2005), pp. 4563-4577.

[44] H. Lin, Z. Zhang, M.Q. Zhang, B. MA, AND M. Li, ZOOM! Zillions of oligos mapped, Bioinformatics, 24:21(2008), pp. 2431-2437.

[45] M. Nei and S. Kumar. Molecular evolution and phylogenetics. New York: Oxford University Press, 2000.

[46] M. Burset And R. Guigo. Evaluation of gene structure prediction programs. Genomics. 34:3(1996), pp. 353-367.

[47] T. Cover and J. Thomas. Elements of information theory. New York: John Wiley \& Sons, Inc. 1991.

[48] L. Zhou, A. Delcher, M. Pertea, and L. Florea. Sim4cc - A cross-species cDNA-to-genome alignment tool. Nucleic Acids Res. 37:11(2009), pp. e80.

[49] HomoloGene. [http://www.ncbi.nlm.nih.gov/sites/entrez?db=homologene]. 
